\title{
Genomic CGH-assessed structural DNA alterations in rectal carcinoma as related to local recurrence following primary operation for cure
}

\author{
K. KODEDA ${ }^{1}$, A. GUSTAFSSON ASTING ${ }^{2}$, C. LÖNNROTH ${ }^{2}$, K. DERWINGER ${ }^{1}$,

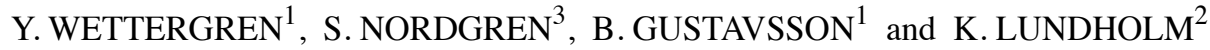 \\ ${ }^{1}$ Campus Östra, Surgical Oncology Laboratory, ${ }^{2}$ Campus Sahlgrenska, Surgical Metabolic Laboratory, \\ ${ }^{3}$ Campus Östra, Colorectal Laboratory, Department of Surgery, The Sahlgrenska Academy at \\ University of Göteborg and Sahlgrenska University Hospital, Göteborg, Sweden
}

Received March 12, 2012; Accepted May 16, 2012

DOI: 10.3892/ijo.2012.1562

\begin{abstract}
Several factors determine overall outcome and possible local recurrence after curative surgery for rectal carcinoma. Surgical performance is usually believed to be the most pertinent factor, followed by adjuvant oncological treatment and tumor histopathology. However, chromosomal instability is common in colorectal cancer and tumor clones are assumed to differ in aggressiveness and potential of causing local recurrence. The aim of this study was, therefore, to evaluate if genetic alterations in primary rectal carcinoma are predictive of local recurrences. A large clinical database with linked bio-bank allowed for careful matching of two patient groups (R0) resected for rectal carcinoma. One group had developed early, isolated local recurrences and the other group seemed cured after 93 months follow-up. DNA from the primary tumors was analysed with array-CGH (comparative genomic hybridization) including 55,000 genomic probes. DNA from all primary tumors in both groups displayed previously reported and well-recognised DNA aberrations in colorectal carcinoma. Significant copy number gains were confirmed in the 4q31.1-31.22 region in DNA from tumors with subsequent local recurrence. Twenty-two affected genes in this region code for products with high relevance in tumor biology (p53 regulation, cell cycle activity, transcription). DNA from rectal carcinoma displayed well-known aberrations as described for colon carcinoma with no obvious prediction of local rectal recurrence. Gains in the 4q31.1-31.22 DNA region are highly potential for local recurrence despite $\mathrm{R} 0$ resection to be confirmed in larger patient materials.
\end{abstract}

Correspondence to: Dr Karl Kodeda, Department of Surgery, Sahlgrenska University Hospital/Campus Östra, S-41685 Göteborg, Sweden

E-mail:karl.kodeda@vgregion.se

Key words: neoplasm recurrence, local rectal neoplasms, array CGH, tumor DNA, HSA4q31.1-31.22

\section{Introduction}

Colorectal cancer is the third most common malignancy in Europe and the USA $(1,2)$. The treatment of patients with rectal cancer has improved significantly the past decades, where meticulous surgery with clear resection margins is important for cure. Besides improved surgery, preoperative radiotherapy and chemoradiotherapy add to improved treatment results. However, local recurrence after resection of rectal cancer is still a substantial clinical problem. Dedicated centers report local recurrence rates in selected series of less than $4 \%$ but population-based results are rather close to $10 \%$ (3-6). Such treatment failures lead to severe, often intractable symptoms and premature death in the majority of patients $(7,8)$. Thus, it is important to gain further knowledge of factors that determine increased risks for local recurrence following primary operation of rectal carcinoma aimed at cure. The present study evaluates whole genomic array-CGH (comparative genomic hybridization) analysis comparing more than 55,000 DNA sites in primary rectal tumors from patients with tissue in a large bio-bank with linked clinical information. DNA from tumors that recurred locally and non-recurrent tumors were analyzed with the aim to link structural DNA-alterations to isolated local recurrence following R0-resections.

\section{Materials and methods}

Selection of patients. The Sahlgrenska University Hospital is a non-profit institution serving a population of approximately 500,000 . All patients with adenocarcinoma of the colon or rectum treated in this institution are prospectively registered in a database with clinopathological variables. Tissue samples are also collected at the time of surgery from all patients operated in office hours. Samples from the tumor and mucosa $>10 \mathrm{~cm}$ from the tumor are collected at the time of specimen extraction, instantly frozen liquid in nitrogen and stored in $-80^{\circ} \mathrm{C}$. The linked database is continuously updated with clinical data such as variables from the pathology report, postoperative oncological treatment and possible recurrent disease. Data are also 


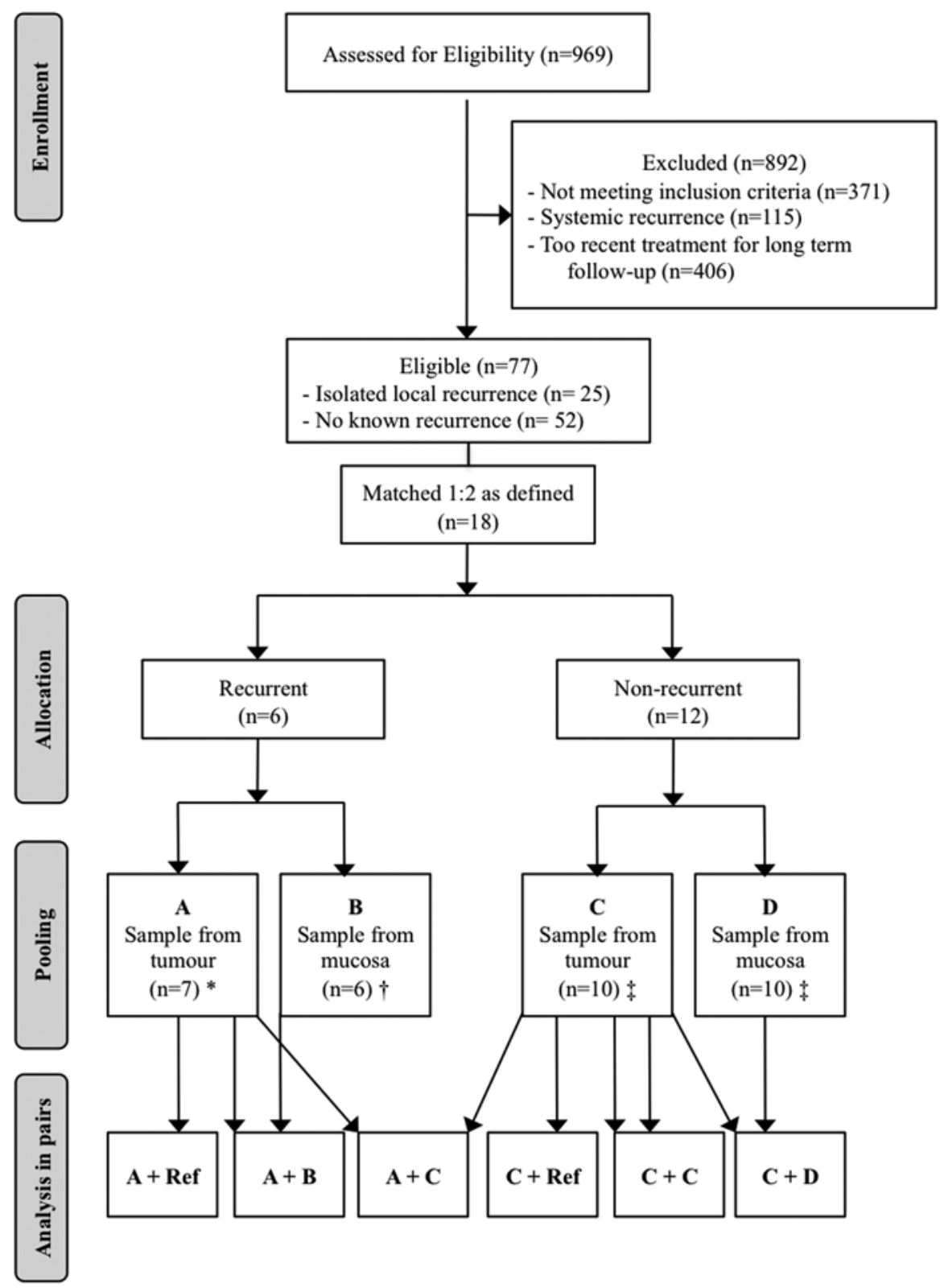

Figure 1. Modified Consort flow diagram. Patients without sign of local or systemic recurrence on long-term follow-up ( $>93$ months) were matched to patients with early locally recurrent disease. Ref denotes a commercially available reference DNA, NA $10851^{*} 8$ (Coriell). "In pool A there were two separate tissue samples from one patient. 'In pool B there were two separate tissue samples from one patient and missing tissue sample from one patient. ${ }^{*}$ Two patients from the non-recurrent group were excluded due to low quality of tissue DNA.

cross-checked with official demographic registries to assure adequate registration of survival.

A total of 2,576 patients were registered in the database during the decade preceding data extraction (January 1999-September 2009). In 969 patients the tumor was located in the rectum. From this cohort two groups of patients were selected for comparative DNA-analysis. Patients in one group were diagnosed with early, isolated local recurrence, while patients in the other group had no sign of local or systemic recurrence at long-term follow-up. Inclusion criteria were: adenocarcinoma in the rectum $(<15 \mathrm{~cm}$ from the anal verge), resectional surgery (anterior resection, abdominoperineal resection, Hartmann's procedure), clear resection margins (R0), and tumor stage I-III [TNM 7 (9)]. Exclusion criteria were: preoperative radiotherapy, distant metastases at the time of surgery and T4-tumors [TNM 7 (9)].
Of 77 eligible patients, 25 had isolated local recurrence and 52 had no sign of recurrent disease on long-term followup. The matched control group (non-recurrent) was stepwise selected on a group characteristics level by selection against study patients (recurrent) for the following variables in order of priority: T-stage, N-stage, differentiation grade, type of surgery, age and gender. Patients with early isolated local recurrence were operated during the years 2002-2006 and the median and mean time to recurrence were 15 and 16 months, respectively. Patients with long-term follow-up were operated from 2002 to 2003 with a minimum time of follow-up of 93 months. See also Consort flow diagram for the study (Fig. 1).

DNA extraction. Samples of tumor and mucosa were retrieved from the bio-bank storage at $-80^{\circ} \mathrm{C}$. Genomic DNA (gDNA) 
was extracted with Qiagen DNeasy Blood and Tissue Kit, cat no. 69504, following mechanical bead-disaggregation of tissue in a TissueLyser (Qiagen) with an RNase treatment included in the method. Concentration and purity of gDNA was measured in a NanoDrop ND-1000A spectrophotometer (NanoDrop Technologies, Inc.). Fractions were separated electrophoretically in $1 \%$ agarose gel for check of quality.

Sample analyses. Genomic DNA was pooled in four groups on basis of origin: tumor from the recurrent group (A), mucosa from the recurrent group (B), tumor from the non-recurrent group (C) and mucosa from the non-recurrent group (D). Pooled gDNA was hybridized in pairs as outlined in Fig. 1. Data analyses from comparisons of DNA from recurrent tumors (A) to DNA from non-recurrent tumors (C) were not regarded as conclusive primary information since copy number gain and loss may cancel each other out. Therefore a commercially available reference DNA was always used as standard DNA in all comparisons, NA 10851*8 (Coriell). Tissue DNA from two patients in the non-recurrent group (C and D) was not possible to analyze due to low DNA quality in tissue specimens resulting in a study population as depicted in Table I.

Labeling and hybridization. Five hundred nanograms of pooled gDNA were labelled with either Cyanine 5-dUTP (test) or Cyanine 3-dUTP (reference) according to the manufacturer's instructions (Agilent Genomic DNA Enzymatic Labeling Kit). Competitive hybridization was performed on Agilent SurePrint G3 Human CGH Microarray Kit 8x60K Oligo, Design ID 021924, according to 'Agilent Oligonucleotide Array-Based CGH for Genomic DNA Analysis. Enzymatic Labelling for Blood, Cells or Tissues' Protocol, version 6.2. Procedure A was chosen for post washes. Images were scanned and quantified on Agilent G2565 AA microarray scanner and fluorescence intensity was extracted using the Feature Extraction (FE, v10.7.1.1) software (Agilent Technologies, USA). At least two of four technical replicates for each pair combination described in Fig. 1 passed the Feature Extraction quality control and were used for analysis. Median spacing of the probes was $33.3 \mathrm{~kb}$ in coding sequences and $78.9 \mathrm{~kb}$ in non-coding sequences of the genome.

Analysis of array data. Dye-normalized, outlier- and background subtracted values were imported into Agilent Genomic Workbench 6.5.0.18 (Agilent Technologies). The result files were filtered at the feature level with Default Feature Filter. Technical replicates were combined and normalized with centralization (treshold 6.0, bin size 10). Aberration analysis was performed with ADM-1 algorithm (treshold 6.0, nesting filter 0 , fuzzy zero on) and filtered with default aberration filter v2 (minimum number of probes 3 , minimum absolute average of $\log$ ratio for region 0.20 ). The statistical confidence interval was $\pm 0.2 \log (2)$ ratio as determined in our earlier study on DNA aberrations in colorectal carcinoma (10). Affected genes in an area of particular interest on chromosome 4 (4q31.1-31.22) were identified with the software algorithm in Genomic Workbench 6.5.0.18 (Agilent Technologies). Identified genes were manually searched for in the NCBI Gene-database and known functions evaluated in relevance to the present context.
Table I. Tumor stage and clinical characteristics of included patients.

\begin{tabular}{|c|c|c|c|}
\hline & $\begin{array}{l}\text { Study patients } \\
\text { (recurrent) } \\
(n=6)\end{array}$ & $\begin{array}{c}\text { Controls } \\
\text { (non-recurrent) } \\
(\mathrm{n}=10)\end{array}$ & p-value \\
\hline \multicolumn{4}{|l|}{ Sex } \\
\hline Female & $3 \quad(50)$ & $5 \quad(50)$ & \\
\hline Male & $3 \quad(50)$ & $5 \quad(50)$ & \\
\hline T-stage & & & 0.87 \\
\hline $\mathrm{T} 1$ & $0 \quad(0)$ & $0 \quad(0)$ & \\
\hline $\mathrm{T} 2$ & $1 \quad(17)$ & $2 \quad(20)$ & \\
\hline $\mathrm{T} 3$ & $5 \quad(83)$ & $8 \quad(80)$ & \\
\hline N-stage & & & 0.92 \\
\hline No & $3 \quad(50)$ & $5 \quad(50)$ & \\
\hline N1 & $2(33)$ & $4 \quad(40)$ & \\
\hline $\mathrm{N} 2$ & $1 \quad(17)$ & $1 \quad(10)$ & \\
\hline \multicolumn{4}{|c|}{ Differentiation, grade } \\
\hline Moderate, G2 & $6(100)$ & $10(100)$ & \\
\hline Poor, G3 & $0 \quad(0)$ & $0 \quad(0)$ & \\
\hline \multirow{2}{*}{\multicolumn{4}{|c|}{$\begin{array}{l}\text { Preoperative } \\
\text { radiotherapy }\end{array}$}} \\
\hline & & & \\
\hline Yes & $0 \quad(0)$ & $0 \quad(0)$ & \\
\hline No & $6(100)$ & $10(100)$ & \\
\hline Operation & & & 0.15 \\
\hline $\mathrm{AR}$ & $2(33)$ & $8 \quad(80)$ & \\
\hline APR & $3 \quad(50)$ & $1 \quad(10)$ & \\
\hline HA & $1 \quad(17)$ & $1 \quad(10)$ & \\
\hline Age (years) & & & 0.97 \\
\hline Mean \pm SD & $74.7 \pm 11.0$ & $74.5 \pm 9.1$ & \\
\hline Median & 74.5 & 78.5 & \\
\hline Range & $58-92$ & $60-84$ & \\
\hline
\end{tabular}

Values in parentheses are percentages. P-values calculated with Pearson's $\chi^{2}$ test except for age where independent samples t-test was used. AR, anterior resection; APR, abdominoperineal resection; HA, Hartmann's procedure.

Confirmation with qPCR. Quantitative real-time polymerase chain reaction (qPCR) was performed on gDNA from individual tumor tissue samples of all studied patients. Primers were chosen to include five areas on chromosome 4 where copy number gain was noted only in the recurrent group (genes SETD7, MGST2, HHIP, SMAD1 and ANAPC10). For comparison, primers were also chosen for analysis of areas on chromosomes 13 and 20 where both patient groups displayed significant copy number gains, with a statistically significant difference between the groups in assay analyses. PCR data were analysed with an efficiency adjusted comparative $\mathrm{Cq}$ method, where a normal DNA area on chromosome 10 served as internal reference. The commercially available reference DNA NA $10851^{*} 8$ (Coriell) was used as standard DNA. 
All primer design was performed with Primer BLAST (http://www.ncbi.nlm.nih.gov/tools/primer-blast/index.cgi? LINK_LOC=BlastHome). Details on primers are presented in Table II. An assay targeting chromosome 10 was already available (TATAA Biocenter, Gothenburg, Sweden). Criteria for good performing assays were: linearity, high efficiency $(>80 \%)$ and negative NTC:s (no template control). All qPCR assays were tested on reference gDNA. For all assays a five-point standard curve was generated with four replicates in each point, run in 5-fold dilution series with primer concentration $200 \mathrm{nM}$ and gDNA template concentrations between $3020-5 \mathrm{pg} / \mu 1$. PCR products from designed assays were analyzed on a $2.2 \%$ FlashGel (Lonza) according to the manufacturer's instructions for control of correct product size and specificity. No unspecific product could be observed in gel analysis (data not shown).

All qPCR analyses were performed with $10 \mu \mathrm{l}$ reaction volume in triplicates on the LightCycler-480 instrument (Roche) in 384 or 96-well plate format using IQ ${ }^{\mathrm{TM}}$ SYBR Green Supermix cat no. 170-8882 (Bio-Rad Laboratories Inc.). Detection was performed in the FAM channel. Template gDNA samples were normalized to $1,000 \mathrm{pg} / \mu 1$ prior to qPCR analysis. All qPCR experiments were performed according to the manufacturer's instructions and all pipetting was performed by robot (EpMotion 5070, Eppendorf, Germany).

Statistical analysis. The statistical confidence interval was set to $\pm 0.2 \log (2)$ ratio in analysis of array-CGH data (10). Independent samples t-test was used to compare $\log (2)$ ratios among groups. Data from qPCR were analysed with the comparative $\mathrm{Cq}$-method. Average Cq-values in groups were analyzed with ANOVA. Individual Cq-values from all assays with technical triplicates were used in analysis of aberrations on chromosome 4. Results are presented as mean and SEM. p-value $<0.05$ was considered statistically significant. Comparison of group characteristics was performed with Pearson's $\chi^{2}$ except for age where independent samples t-test was used.

The bio-bank was instituted and maintained in accordance to national regulations. The present study was approved by the regional ethics review board (Dnr 261-08).

\section{Results}

The selection of 6 patients with early, isolated local recurrence after primary cancer surgery and 12 matched non-recurrent control patients out of 77 eligible patients is outlined in Fig. 1. Study and control patients showed comparable clinical characteristics, although they were selected and matched according to clinical characteristics on group basis (Table I).

Genomic DNA from rectal carcinomas had DNA sequences with statistically significant aberrations compared with reference DNA. As depicted in Fig. 2, several DNA areas were significantly affected in both recurrent and nonrecurrent tumors, assessed by CGH-array analysis $(\mathrm{p}<0.05)$. Many of these affected regions are known to be involved in colorectal carcinogenesis, e.g., on chromosomes 5, 8, 13, 17, 18 and 20 (11-14). However, DNA aberrations seemed overall more pronounced in DNA from non-recurrent tumors. This appeared in both copy number gains (chromosomes 5, 8, 13, 20) and copy number losses (chromosomes 1, 4, 5, 17, 18, 22) (Fig. 2), although, qPCR quantification on sequences in

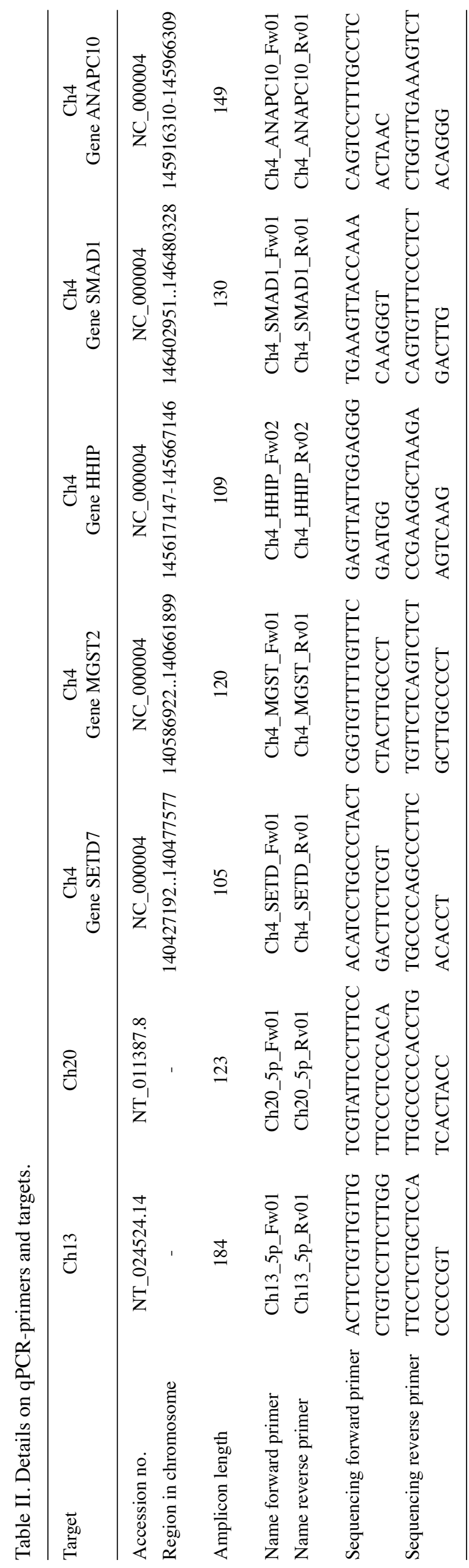




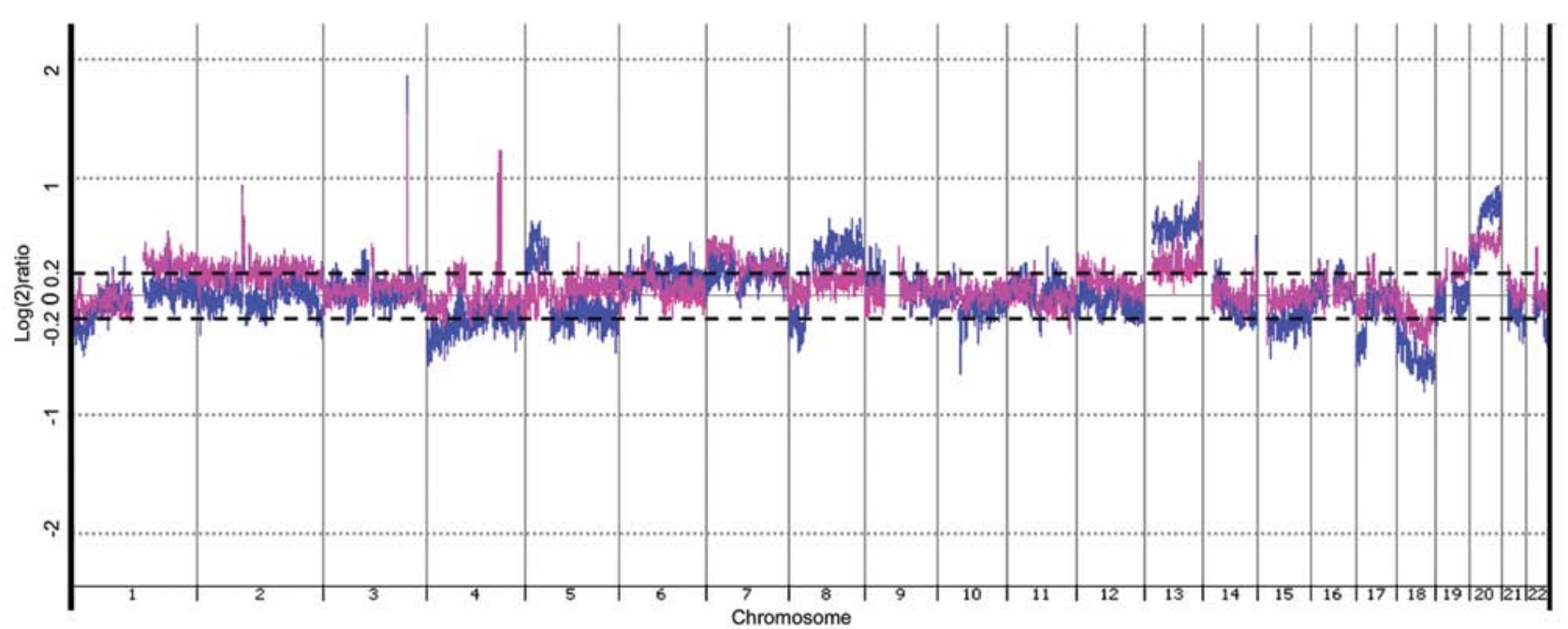

Figure 2. Whole genome analysis with array-CGH, as described in Materials and methods. DNA from patients vs. reference DNA. Pink, locally recurrent tumors. Blue, non-recurrent tumors. Positive values signifies copy number gain and negative values copy number loss. The $95 \%$ statistical confidence interval was \pm 0.2 $\log (2)$ ratio.

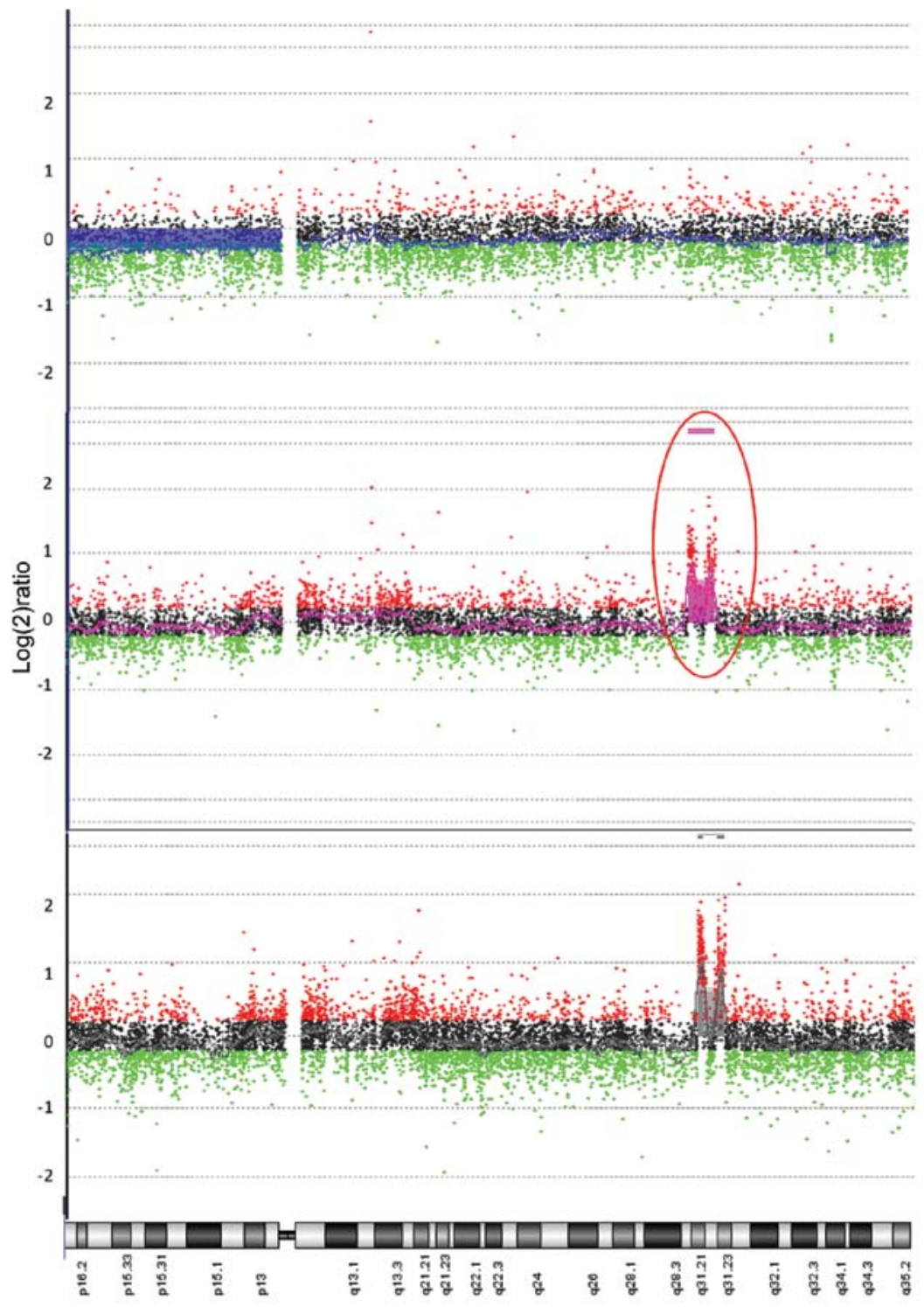

Figure 3. Chromosome 4 analysed with array-CGH. 4q31.1-31.22 highlighted. Positive values (red) signify copy number gain and negative values (green) copy number loss. Central values (black) are within the $95 \%$ statistical confidence interval $\pm 0.2 \log (2)$ ratio. Top, tumor tissue DNA from non-recurrent group vs. reference DNA. Middle, tumor tissue DNA from recurrent group vs. reference DNA. Bottom, tumor tissue DNA vs. mucosa DNA from patients with local recurrence. 
Table III. Genes located in the 4q31.1-31.22 region with examples of known function.

\begin{tabular}{|c|c|}
\hline Gene & Known function \\
\hline SETD7 & Involved in p53 regulation \\
\hline MGST2 & Involved in production of leukotrienes and PGE2 \\
\hline MAML3 & Mastermind-like 3, involved in regulation of Notch signalling \\
\hline SCOC & Interacts with ADP-ribosylation factor-like proteins \\
\hline CLGN & May play a role in spermatogenesis and infertility \\
\hline ELMOD2 & Antiviral response \\
\hline $\mathrm{UCP} 1$ & Only expressed in brown adipose tissue \\
\hline TBC1D9 (MDR1) & $\begin{array}{l}\text { Transmembrane efflux pump, decreased intracellular accumulation and multidrug resistance. MDR1-negative } \\
\text { samples were most common among tumor types known to be relatively responsive to chemotherapy }\end{array}$ \\
\hline RNF150 & Ring finger protein \\
\hline ZNF330 & Zinc finger protein \\
\hline GAB1 & $\begin{array}{l}\text { Important mediator of branching tubulogenesis and plays a central role in cellular growth response, } \\
\text { transformation and apoptosis }\end{array}$ \\
\hline SMARCA5 & Regulate transcription \\
\hline LOC441046 & Pseudogene \\
\hline GYPE & Glycophorin E, M blood group antigen \\
\hline GYPB & Glycophorin B, M blood group antigen \\
\hline GYPA & Glycophorin A, M blood group antigen \\
\hline HHIP & Inhibitor of Hedgehog signalling, involved in developmental process, implicated in COPD and lung cancer \\
\hline ANAPC10 & Progression through cell cycle \\
\hline ABCE1 & Transport across extra- and intra-cellular membranes \\
\hline OTUD4 & Expressed at HIV-1 infection \\
\hline SMAD1 & $\begin{array}{l}\text { Signal transducer and transcriptional modulator in for example cell growth, apoptosis, immune responses, } \\
\text { morphogenesis and development }\end{array}$ \\
\hline MMAA & Translocation of cobalamin in mitochondria \\
\hline
\end{tabular}

Table IV. Quantitative PCR analyses on gDNA from recurrent and non-recurrent individual tumors.

\begin{tabular}{lcccccccc}
\hline & Ch13 & Ch20 & Ch4 & Ch4 & Ch4 & Ch4 & Ch4 & $\begin{array}{c}\text { Ch4 } \\
\text { SETD7 }\end{array}$ \\
& $5 \mathrm{p} 01$ & $5 \mathrm{p} 01$ & MGS2 & HHIP & SMAD1 & ANAPC10 & Sum of all probes \\
\hline Recurrent (n=7) & $1.16 \pm 0.13$ & $1.33 \pm 0.15$ & $2.05 \pm 1.13$ & $1.13 \pm 0.61$ & $0.92 \pm 0.07$ & $2.12 \pm 1.19$ & $1.71 \pm 0.83$ & $1.62 \pm 0.37$ \\
Non-recurrent (n=10) & $1.24 \pm 0.21$ & $1.23 \pm 0.13$ & $1.04 \pm 0.19$ & $0.64 \pm 0.04$ & $0.78 \pm 0.04$ & $0.91 \pm 0.06$ & $0.82 \pm 0.05$ & $0.84 \pm 0.04$ \\
p-value $^{\mathrm{a}}$ & $\mathrm{ns}$ & $\mathrm{ns}$ & $\mathrm{ns}$ & $\mathrm{ns}$ & $\mathrm{ns}$ & $\mathrm{ns}$ & $\mathrm{ns}$ & 0.0147 \\
\hline
\end{tabular}

Efficiency adjusted $\Delta \Delta$ Cq-values. Mean \pm SEM. ns, not statistically significant. Details on primers are outlined in Table II. ${ }^{\text {a ANOVA. }}$

chromosomes 13 and 20 did not confirm statistically different alterations between the groups (Table IV).

In contrast, an aberration on chromosome 4 with great magnitude was noted in DNA from tumors in the locally recurrent group. This gain on chromosome 4 (4q31.1-31.22) was thus detected in DNA from locally recurrent tumors when compared to reference DNA, $\mathrm{p}<0.0001$, with no such alterations in the non-recurrent group compared to reference DNA (Figs. 2 and 3). The difference in copy number DNA between groups in this region on chromosome 4 was also statistically significant when comparing tumor tissue versus mucosa from patients with locally recurrent disease. This indicates a genotype specific alteration in tumor DNA and not in host DNA, p<0.0001 (Fig. 3).

Detailed analysis of relevant regions on chromosome 4 showed that it contained over 100 genes, of which 22 were significantly affected. These 22 genes encode proteins with several known functions including p53 regulation, progres- 
sion through cell cycle as well as regulation of transcription (Table III).

Confirmation with quantitative real-time PCR of gDNA from individual tumor tissue samples including all studied DNA probes showed a statistically significant difference between recurrent and non-recurrent tumors targeting the region of interest on chromosome 4 , which analytically confirms the significant array-CGH findings on a patient group level (Table IV).

\section{Discussion}

Local recurrence after rectal cancer surgery is a main quality indicator in the management of patients with rectal cancer. It is well established that surgical technique is of crucial importance and the concept of TME (total mesorectal excision) is today universally accepted. Radiotherapy, especially preoperatively, and in selected cases preoperative chemoradiotherapy, can further improve the clinical outcome. However, as Marijnen et al has pointed out (15), radiotherapy cannot compensate for positive tumor resection margins. Inability to achieve clear resection margins (R0) entails such a high risk of local recurrence that many surgeons regard this as persistent rather than recurrent disease. Established and suggested risk factors for local recurrence related to the surgical quality include inadequate distal margin, circumferential resection margin $<1 \mathrm{~mm}$, perioperative perforation of the rectum, inadequate clearance of intraluminal viable tumor cells before transection of the bowel, breaching of the mesorectal fascia and producing a non-cylindrical specimen in abdominoperineal resection. Other risk factors include tumor size, stage, location, grade of differentiation, tumor budding, and lymphatic, perineural or vascular invasion. Thus, with this knowledge in mind, stepwise selection of study and control patients (1:2) retrieved two groups with comparable clinical characteristics for DNA analysis.

Despite recent advances in the management of rectal cancer patients, local recurrences do occur. Dedicated centers report local recurrence rates below $4 \%$ in selected series (3-5) and population-based recurrence rates of 7-9\% are not uncommon (6). The local recurrence rate in the present population-based cohort is comparable to the national average for the relevant period, i.e., less than $10 \%$. This included all patients with a curative or palliative resections. Approximately $45 \%$ of patients in our database were operated with anterior resection, $30 \%$ with abdominoperineal resection, $10 \%$ Hartmann's procedure and $10 \%$ with local excision or TEM (transanal endoscopic microsurgery). Thus, the cohort in our database appears relevant in general perspectives of rectal cancer surgery. Although, our present material represents low statistical power, it is important to know that recruiting a double number of patients, would require 15-20 years continuous collection of tissue samples from all patients operated in the largest colorectal department in Scandinavia. The main reasons for this is declining local recurrence rates and increasing proportions of patients treated with preoperative radiotherapy.

The importance of 'lateral' lymph nodes in the context of local recurrence is unclear. Our present material appears well matched relative to lymph node metastases encountered during pathological examination of the operative specimens. The presence of remaining tumor tissue after rectal surgery may not be enough for a local recurrence to be established. This conclusion is supported by the fact that not all patients with R1-resections (microscopically involved resection margin) develop local recurrences $(16,17)$. Also, not all patients in whom the operative field has been contaminated by tumor cells, due to perioperative perforation of the tumor, will develop local recurrences (18). Furthermore, not all patients develop anastomotic recurrence despite the fact that there are viable cells in the lumen of the bowel following resection (19). Thus it appears, there are both known and unknown factors that determine the patients who will experience local recurrences. Such factors may be host- and immune-related, or primarily related to tumor cell biology. Therefore, efforts have also been made to identify risk factors at tumor cell level but such potential findings remain to be confirmed and clinically applied in rectal cancer treatment $(16,17)$. Possibly, there are rectal tumors that are more prone to recur locally than others and such characteristics may thus be related to aberrations in the tumor genome. It has been proposed that colorectal adenocarcinomas can be divided in at least five subgroups based on genetic characteristics $(12,20,21)$. Our previous studies with array-CGH have also shown that there are aberrations in the tumor genome of colorectal malignancies that are stage-dependent (14). It is also well recognized that tumors are heterogeneous and contain several clones and perhaps different cancer stem cells $(13,21)$. By evolutionary clonal selection some clones may prevail (13). Thus, it is likely that different tumors should vary in characteristics by several clinical aspects such as ability to metastasize and generate local recurrence.

Accordingly, genomic DNA from both recurrent and non-recurrent rectal tumors in the present study showed aberrations in DNA areas that have previously been described to be frequently involved in colorectal carcinogenesis [e.g., on chromosomes 5, 8, 13, 17, 18 and 20 (11-14)]. Interestingly, the copy number gains and losses were generally less pronounced in the present tumors prone to local recurrence; an observation which may not be expected in the light of numerous reports on the relationship between copy number gains and poor prognosis in colon carcinomas. Again, such observations may point to the possibility that systemic spread and local recurrent growth may indicate different genetic background. Thus, several wellrecognized DNA alterations are unlikely of major importance for the development of local recurrences in rectal carcinoma following R0 resections.

The area on chromosome 4 (4q31.1-31.22), where locally recurrent tumors displayed significant copy number gains compared to non-recurrent tumors, has not been reported earlier in colorectal cancer. A similar gain pattern in comparison of tumor DNA to mucosa DNA in recurrent patients supports that observed DNA alterations were genotype specific for tumor cells and not a patient DNA phenomenon, even though low statistical power in our present material should exclude firm conclusions. However, affected genes located in the DNA area on chromosome 4 code for at least 22 products, some of which have known functions that are of high relevance in tumor biology, such as regulation of p53, cellular growth, cellular transformation, progression through cell cycle, regulation of apoptosis as well as arachnoid acid metabolism with production of leukotriens and prostaglandins. Whether few or several genes in this area act in concert to promote recurrent growth must await future research. 
In conclusion, the availability of a large bio-bank on rectal carcinoma with linked clinopathological data was a prerequisite for careful matching of groups of non-irradiated patients and subsequent evaluation of possible genetic differences between locally recurrent and non-recurrent rectal carcinomas. The surgical management and clinical outcome in our study population was in agreement with international standards. Most well recognized aberrations in colorectal carcionogenesis were identified in both recurrent and non-recurrent tumors, but seemed to be of less importance for local recurrences. However, the $4 \mathrm{q} 31.1-31.22$ region represents a potential area for further investigations in larger patient cohorts.

\section{Acknowledgements}

This work was supported by grants from the Swedish Cancer Society, the Swedish State under the LUA/ALF agreement, the Göteborg Medical Society and the Anna-Lisa and Bror Björnsson Foundation. We acknowledge the expert technical skill of Ingrid Palmgren, Marianne Åkerström, Marianne Andersson and Jacqueline Flach. We thank Hillevi Björkqvist and Ann-Louise Helminen for handling surgical samples and Lena Munro and Birgitta Sjöberg for excellent administration of the clinical database. The authors are not aware of any affiliations, memberships, funding or financial holdings that might be perceived as affecting the objectivity of this paper.

\section{References}

1. European Cancer Observatory (ECO): Cancer fact sheet 2011 http://eu-cancer.iarc.fr/country-930-european-union-27.html,en. [Accessed: 2011, Nov 8].

2. American Cancer Society: Cancer Facts and Figures 2011. http:// www.cancer.org/acs/groups/content/@epidemiologysurveilance/ documents/document/acspc-029771.pdf. [Accessed: 2011, Nov 8]

3. Edwards DP, Sexton R, Heald RJ and Moran BJ: Long-term results show triple stapling facilitates safe low colorectal and coloanal anastomosis and is associated with low rates of local recurrence after anterior resection for rectal cancer. Tech Coloproctol 11: 17-21, 2007.

4. MacFarlane JK, Ryall RD and Heald RJ: Mesorectal excision for rectal cancer. Lancet 341: 457-460, 1993.
5. Moore E, Heald RJ, Cecil TD, Sharpe GD, Sexton R and Moran BJ: Almost all five year disease free survivors are cured following rectal cancer surgery, but longer term follow-up detects some late local and systemic recurrences. Colorectal Dis 7: 403-405, 2005

6. Pahlman L, Bohe M, Cedermark B, et al: The Swedish rectal cancer registry. Br J Surg 94: 1285-1292, 2007.

7. Camilleri-Brennan J and Steele RJ: The impact of recurrent rectal cancer on quality of life. Eur J Surg Oncol 27: 349-353, 2001.

8. Palmer G, Martling A, Cedermark B and Holm T: A populationbased study on the management and outcome in patients with locally recurrent rectal cancer. Ann Surg Oncol 14: 447-454, 2007.

9. Sobin L, Gospodarowicz M and Wittekind C: TNM Classification of Malignant Tumours. 7th edition. Wiley-Blackwell, 2010.

10. Lagerstedt KK, Staaf J, Jonsson G, et al: Tumor genome wide DNA alterations assessed by array CGH in patients with poor and excellent survival following operation for colorectal cancer. Cancer Inform 3: 341-355, 2007.

11. Brenner BM and Rosenberg D: High-throughput SNP/CGH approaches for the analysis of genomic instability in colorectal cancer. Mutat Res 693: 46-52, 2010.

12. Cunningham D, Atkin W, Lenz HJ, et al: Colorectal cancer. Lancet 375: 1030-1047, 2010.

13. Fearon ER: Molecular genetics of colorectal cancer. Annu Rev Pathol 6: 479-507, 2011

14. Lagerstedt KK, Kristiansson E, Lonnroth C, et al: Genes with relevance for early to late progression of colon carcinoma based on combined genomic and transcriptomic information from the same patients. Cancer Inform 9: 79-91, 2010.

15. Marijnen CA, Nagtegaal ID, Kapiteijn E, et al: Radiotherapy does not compensate for positive resection margins in rectal cancer patients: report of a multicenter randomized trial. Int J Radiat Oncol Biol Phys 55: 1311-1320, 2003.

16. Enriquez-Navascues JM, Borda N, Lizerazu A, et al: Patterns of local recurrence in rectal cancer after a multidisciplinary approach. World J Gastroenterol 17: 1674-1684, 2011.

17. Den Dulk M, Marijnen CA, Putter H, et al: Risk factors for adverse outcome in patients with rectal cancer treated with an abdominoperineal resection in the total mesorectal excision trial. Ann Surg 246: 83-90, 2007.

18. Jorgren F, Johansson R, Damber L and Lindmark G: Oncological outcome after incidental perforation in radical rectal cancer surgery. Int J Colorectal Dis 25: 731-740, 2010.

19. Kodeda K, Holmberg E, Jorgren F, Nordgren S and Lindmark G: Rectal washout and local recurrence of cancer after anterior resection. Br J Surg 97: 1589-1597, 2010.

20. Jass JR: Classification of colorectal cancer based on correlation of clinical, morphological and molecular features. Histopathology 50: 113-130, 2007

21. Markowitz SD and Bertagnolli MM: Molecular origins of cancer: molecular basis of colorectal cancer. N Engl J Med 361: 2449-2460, 2009. 